\title{
Mediating macrophage immunity with wogonin in mice with vascular inflammation
}

\author{
JINGWEI WANG ${ }^{1,2^{*}}$, KUNXIA LI $^{3^{*}}$, YUPENG LI ${ }^{4}$ and YULIN WANG ${ }^{1}$ \\ ${ }^{1}$ Department of Paediatrics, Shandong Provincial Hospital Affiliated to Shandong University, Jinan, Shandong 250033; \\ ${ }^{2}$ Department of Paediatrics, Yantaishan Hospital, Yantai, Shandong 264001; ${ }^{3}$ Department of Paediatrics, \\ Yantai Yuhuangding Hospital, Yantai, Shandong 264000; ${ }^{4}$ Department of \\ Paediatrics, Rizhao People's Hospital, Rizhao, Shandong 276800, P.R. China
}

Received February 2, 2017; Accepted August 2, 2017

DOI: $10.3892 / \mathrm{mmr} .2017 .7611$

\begin{abstract}
Vascular inflammation may induce a number of diseases, including organ damage or failure, heart attack and stroke. The present study aimed to investigate the use of wogonin, a compound extracted from herbs, to mediate inflammatory reactions in vascular inflammation. Wogonin was loaded in a well-characterized polymeric biomaterial carrier. In mice with streptozotocin-induced vascular inflammation, wogonin treatment regulated the production of inflammatory cytokines, including interleukin-6, tumor necrosis factor- $\alpha$ and granulocyte macrophage colony-stimulating factor. To understand the impact of wogonin on major immune cells, macrophages were treated with wogonin in vitro. It was determined that wogonin did not affect macrophage viability, and that wogonin regulated the relative ratio of M1 versus M2 macrophages. In addition, in co-culture, wogonin decreased inflammatory cytokine production and regulated the activation of macrophage surface markers including CD80, CD86 and CD40. Results from the present study may aid in our understanding of the effects of wogonin in regulating inflammation, especially its effects on macrophages.
\end{abstract}

\section{Introduction}

Vascular inflammation is a severe complication in many countries around the world. This condition requires attention as it is usually associated with other diseases, including diabetes, hypertension and lipid disorders $(1,2)$. In the clinic, vascular

Correspondence to: Dr Yulin Wang, Department of Paediatrics, Shandong Provincial Hospital Affiliated to Shandong University, 324 Jingwuweiqi Road, Huaiyin, Jinan, Shandong 250033, P.R. China

E-mail:wangyulin07@sina.com

*Contributed equally

Key words: vascular inflammation, wogonin, macrophage, cytokines, surface markers inflammation may also accompany endothelial dysfunctions, and may lead to cardiovascular diseases over time. In a typical situation, inflammatory responses usually begin in the vascular system or vascular endothelium and may lead to endothelial cell dysfunction, which is one of the main causes for cardiovascular diseases $(3,4)$. With the cascade of vascular inflammation, endothelial cells express inflammatory cytokines that recruit immune cells (for example, macrophages and leukocytes) to the inflammatory sites, thereby amplifying the inflammatory response that may lead to subsequent tissue injuries $(2,5)$.

Wogonin (5,7-dihydroxy-8-methoxyflavone) is a flavonoid-like chemical compound that received extensive attention in recent years owing to its numerous potential therapeutic effects (6). Wogonin is extracted from Scutellaria baicalensis Georgi, and previous studies have reported that this compound may inhibit cancer progression and reduce the production of oxidants in cells and tissues (7-11). For example, wogonin may inhibit proliferation in several cancer cell lines, including hepatocellular carcinoma, ovarian and promyeloleukemic cancer cells (12-16). In anti-oxidative applications, wogonin decreased nitric oxide (NO) production in macrophage cells that were stimulated with lipopolysaccharide (LPS) (17). In another study, wogonin inhibited hydrogen peroxide-induced oxidative stress in SH-SY5Y neuroblastoma cells (18). Similar studies also demonstrated the antioxidant and free radical scavenging effects of wogonin as well as its analogues such as baicalein (19).

The immune modulatory effects of wogonin in different in vitro and in vivo disease models have been reported previously $(10-12,17)$. In one study, it was reported that wogonin upregulated immunoglobulin A levels in mice with colitis (20). The study also reported that wogonin downregulated the concentration levels of interleukin (IL)-4, IL-5 and IL-10 cytokines in mice. Thus, this study demonstrated that wogonin may be able to alleviate the inflammatory response in mice with colitis through $\mathrm{T}$ helper 2 cell responses. In another study, wogonin inhibited the inflammation induced by toll-like receptor 4 adjuvants, such as LPS, in root ganglion neurons by downregulating the nuclear factor- $\kappa \mathrm{B}$ and mitogen-activated protein kinase signaling pathways $(7,10)$. These previous studies also revealed that wogonin suppressed 
the secretion of pro-inflammatory mediators, including cyclooxygenase (COX)-2, inducible NO synthases, IL-1 $\beta$, IL-6 and tumor necrosis factor- $\alpha$ (TNF- $\alpha)(7,10)$. Other studies have reported that wogonin may inhibit the production of $\mathrm{NO}$, prostaglandin E2, monocyte chemoattractant protein-1 and COX-2, all of which are indicators of inflammation in cells or tissues $(11,20,21)$. The anti-inflammatory functions of wogonin have also been demonstrated in several in vivo animal models, such as mouse colitis model, mouse skin inflammation model and LPS-induced mouse lung injury model (20-22). The present study examined the effects of wogonin in modulating the inflammatory immune responses in vascular inflammation. To facilitate the strategic delivery and release of wogonin, this therapeutic component was encapsulated into a biodegradable carrier, poly (lactic-co-glycolic) acid (PLGA), a biocompatible material approved by the US Food and Drug Administration (FDA).

\section{Materials and methods}

Ethics statement. All the experiments that involve the use of animals were approved by the Animal Care and Use Committee in Shandong Provincial Hospital Affiliated to Shandong University (Jinan, China), and followed the institute's regulations and the local and governmental laws on animal care and protection.

Materials. Bacterial LPS (from Escherichia coli O55:B5) was purchased from Sigma-Aldrich (Merck KGaA, Darmstadt, Germany). RPMI-1640 cell culture medium was purchased from VWR International (Leicestershire, UK). Cell culture supplements, including fetal bovine serum FBS, penicillin and streptomycin were purchased from Thermo Fisher Scientific, Inc. (Waltham, MA, USA). PLGA (lactide:glycolide, 50:50; MW, 30,000-60,000) was from Sigma-Aldrich (Merck KGaA).

Cell culture. Macrophages were raised in RMPI-1640 medium supplemented with $10 \%$ FBS, $100 \mathrm{U} / \mathrm{ml}$ penicillin and $100 \mathrm{mg} / \mathrm{ml}$ streptomycin. The cells were cultured in an environment enriched with $5 \% \mathrm{CO}_{2}$ at $37^{\circ} \mathrm{C}$ at a density of $4 \times 10^{5}$ cells per petri dish ( 4 in. plastic petri dishes), and maintained with a certain degree of humidity by adding water in the bottom layer of a cell culture incubator. For the viability study, cells from mice with no treatment were used as a control (CTRL). Cells from mice induced with vascular inflammation were used as a negative control. These two groups of cells received no wogonin treatment. In other three groups, cells were treated with different dose of wogonin $(10,25$ and $100 \mu \mathrm{g} / \mathrm{ml})$, either in soluble form or carried in the biomaterial carrier. The cells were treated for 48 hours before being tested for viability. For soluble wogonin, the wogonin cargo was added into PBS buffer and used for in vitro and in vivo.

Animal experiments. A total of 32 female DBA/1J mice (age, 5-8 weeks, $\sim 18 \mathrm{~g}$ each) were obtained from The Animal Center at Shandong University. Streptozotocin was used to induce the disease model with the method established and reported previously $(22,23,24)$. All the mice were raised in a room with a 12-h light/dark cycle. Temperature of the room was kept at $23^{\circ} \mathrm{C}$ and $47 \%$ humidity, with free access to food and water for all the mice. For the in vivo cytokines study, the mice were divided randomly into 4 groups (8 mice/group): Mice with no treatment (i.e., No treat), mice induced with LPS $(1 \mathrm{mg} / \mathrm{kg})$ daily for 3 days (Diseased), mice treated with soluble wogonin (275 $\mu \mathrm{g}$ wogonin per mouse; Soluble) and mice treated with materials carrying wogonin daily (Wog/NP). The injections were performed via tail vein. Mice were anesthetized by using ketamine and medetomidine (50 and $1 \mathrm{mg} / \mathrm{kg}$, respectively) if required.

Synthesis and characterization of cargo loading nanoparticles. A previously described emulsion method was used to synthesize nanoparticles to carry wogonin (25). Briefly, wogonin (3 mg; cat. no. 102987-206; VWR, Radnor, PA, USA) and PLGA polymer $(20 \mathrm{mg})$ were dissolved together in the dichloromethane (DCM) solvent at room temperature. The mixture was sonicated for $1 \mathrm{~min}$ to make a monodispersed solution on ice. The mixture was then added into $5 \mathrm{ml}$ polyvinyl alcohol (PVA) solution in water $(2 \% \mathrm{w} / \mathrm{w})$. Following sonication for $20 \mathrm{~min}$ on ice, the mixture of aqueous solution and organic solvent was stirred with a magnetic bar for $90 \mathrm{~min}$ at $750 \mathrm{rpm}$. Following the reaction, the particles were centrifuged at $6,200 \mathrm{x} \mathrm{g}$ for $15 \mathrm{~min}$ for collection at $4^{\circ} \mathrm{C}$. Deionized water was used to wash the particles twice, followed by lyophilizing the particles to obtain dry powder by using a freeze dryer (Labconco, Kansas City, MO, USA). The particles were stored at $-20^{\circ} \mathrm{C}$ until use. A JEOL-JSM $7500 \mathrm{~F}$ Scanning Electron Microscope (SEM; JEOL Ltd., Tokyo, Japan) was used to analyze the particle morphology and demonstrate particle size. Particle size was also measured by dynamic light scattering with a Zetasizer Nano ZS system (Malvern Instruments, Ltd., Malvern, UK). Samples for SEM characterization were collected by dropping $50 \mu \mathrm{l}$ particles $(200 \mu \mathrm{g} / \mathrm{ml}$ in DI water) onto a glass substrate, and the glass substrate with particles were coated with a thin layer of gold (Emitech K550X; Energy Beam Sciences Inc., CT, USA; 45 sec coating). During particle size assessment, the incident beam was scattered at $90^{\circ}$, and each measurement was taken 3 times to obtain an average value.

Enzyme-linked immunosorbent assay (ELISA) assays. ELISA was performed according to the manufacturer's protocols. Briefly, 75-90 $\mu 1$ peripheral blood was collected from mice 3 days following treatment. The blood was maintained on ice; serum was obtained by centrifugation at 20,000 x $\mathrm{g}$ for $5 \mathrm{~min}$ at $4^{\circ} \mathrm{C}$. Serum was stored at $-80^{\circ} \mathrm{C}$ prior to use to maintain its bioactivity. The expression levels of IL-6 (cat. no. KMC0061; Thermo Fisher Scientific, Inc.), TNF- $\alpha$ (cat. no. KMC3011; Thermo Fisher Scientific, Inc.) and granulocyte macrophage colony-stimulating factor (GM-CSF; cat. no. ab46078; Abcam, Cambridge, UK) were measured. To test the level of cytokines secreted by macrophages, the cells were treated with different formulations for $60 \mathrm{~h}$ and then tested with ELISA kit by following the manufacturer's protocols.

Macrophage viability measurement. The effects of the particles and the cargo (Wog./NP) on cell viability were measured by co-culturing macrophages with soluble wogonin or wogonin carried in the nanoparticles (Wog./NP). Macrophages were collected from bone marrow as previously described $(26,27)$. 
The cells with different treatments (i.e., macrophages with no treatment, macrophages with 10,25 and $100 \mu \mathrm{g} / \mathrm{ml}$, respectively) were cultured for $48 \mathrm{~h}$ and then collected for analysis. Cells were cultured with $5 \% \mathrm{CO}_{2}$ at $37^{\circ} \mathrm{C}$ at a density of $1 \times 10^{5}$ cells per well in a 96-well plate. Following two washes with PBS, the cells were stained with trypan blue $(1: 1,000$ dilution in PBS) for viability test; the number of viable cells was measured with a NanoEnTek Automated Cell Counter (NanoEnTek, Inc., Seoul, Korea).

Cell staining and flow cytometry. Macrophages were treated for $24 \mathrm{~h}$; cells were collected from 96 well plates $\left(2 \times 10^{5}\right.$ per well) and washed with PBS twice. Subsequently, cells were treated with Fc-block to reduce non-specific binding during the staining. The cells were stained with the following fluorescence-labeled antibodies for $25 \mathrm{~min}$ at room temperature: Fluorescein isothiocyanate-conjugated CD80 (1:200 in PBS plus 1\% BSA, cat. no. 561954; BD Biosciences, Franklin Lakes, NJ, USA); phycoerythrin-conjugated CD86 (553692; 1:200; BD Biosciences); and allophycocyanin-conjugated CD40 (124610; BioLegend, Inc., San Diego, CA, USA). All the antibodies were used at 1:200 dilution in PBS. Following antibody staining, the cells were collected and washed with PBS for $1 \mathrm{~min}$ to remove non-specific staining. To analyze the relative ratio of M1 and M2 macrophages, CD80 and CD86 was selected as M1 macrophage markers and were stained with fluorescein isothiocyanate-conjugated CD80 (561954; 1:200; BD Biosciences, Franklin Lakes, NJ, USA); phycoerythrin-conjugated CD86 (553692; 1:200; BD Biosciences); and allophycocyanin-conjugated CD40 (1:200; BioLegend, Inc.). CD71 and CD206 were selected as M2 macrophage surface markers. These two markers were stained with monoclonal antibody [R17217 (RI7 217.1.4), APC labeled, eBioscience; Thermo Fisher Scientific, Inc.] and CD206 (MMR) monoclonal antibody (MR6F3; PE labeled; both at 1:200; eBioscience; Thermo Fisher Scientific, Inc.), respectively. The cells were dispersed and counterstained in PBS with $0.1 \%$ DAPI at room temperature and analyzed by flow cytometry for $45 \mathrm{~min}$. FlowJo version 10.1 (FlowJo LLC, Ashland, OR, USA) was used to analyze the macrophage staining.

Statistical analysis. Statistical analysis was conducted with analysis of variance (ANOVA) to determine the statistical difference between different groups. If ANOVA indicated a significant difference, then a t-test with Tukey's correction for multiple comparisons was employed to examine the individual differences. GraphPad Prism version 5.0 (GraphPad Software, Inc., La Jolla, CA, USA) was used for analysis. The data are presented as the mean \pm standard error of the mean. $\mathrm{P}<0.05$ was considered to indicate a statistically significant difference.

\section{Results and Discussion}

For a complete understanding of the study, the chemical structure of wogonin is provided (Fig. 1A). Wogonin was loaded into a biocompatible carrier, PLGA; the polymer was produced by an emulsion method, which generated spherical PLGA particles that carried wogonin. SEM imaging demonstrated that the particles had a spherical shape (Fig. 1B), and dynamic light scattering indicated that the particles had a diameter of $107 \pm 39 \mathrm{~nm}$ (Fig. 1C). This nanoscale size of the particles is appropriate for in vivo usage, and the loading level of wogonin within the particles was assessed by dissolving the Wog./NP powder in $\mathrm{NaOH}(3 \mathrm{M})$ and measuring the absorbance at $275 \mathrm{~nm}$. The loading of wogonin in the nanoparticles was $\sim 12.6 \mu \mathrm{g} / \mathrm{mg}$ PLGA. These particles were used in subsequent in vivo experiments to examine their effects on controlling inflammatory responses. PLGA is an ideal material for delivering immune and therapeutic cargos. The advantages of using PLGA as a delivery vehicle include co-delivery of different cargo, targeting of specific tissues or cells and controlled release. In a previous study, the inflammatory function of PLGA to the immune system has been reported to be minor (26); several additional studies demonstrated that PLGA microparticles, instead of PLGA nanoparticles, were able to promote the production of inflammatory cytokines such as TNF- $\alpha(28,29)$. However, considering that PLGA is one of several existing materials approved by the FDA for use in humans, and that PLGA at the nanoscale level does not induce notable inflammation in vitro (29), this material was used in the present study to facilitate the delivery of wogonin.

The effects of wogonin were examined in vivo, using both a soluble form and one carried in PLGA. Mice with streptozotocin-induced vascular inflammation were treated with $275 \mu \mathrm{g}$ wogonin daily. Peripheral blood was collected from mice 3 days post-treatment and analyzed for the levels of cytokines in the serum, including IL-6, TNF- $\alpha$ and GM-CSF (Figs. 2A-C, respectively). The mice with vascular inflammation exhibited a higher level of inflammatory cytokines in their serum compared with mice in the other groups. The two wogonin treatments both reduced the production of IL-6 cytokines in mice with vascular inflammation. Compared with the soluble formulation, PLGA-carried wogonin appeared to be more effective in reducing IL-6 production (Fig. 2A), probably because the nanoparticles improved the bioavailability of wogonin. Wogonin treatment, either in a soluble form or carried in PLGA nanoparticles, had a slight effect in reducing the production of TNF- $\alpha$, and no statistically significant difference was identified between the soluble and nanoparticle forms (Fig. 2B). For GM-CSF production, a similar trend as IL-6 level was observed; that is, both wogonin treatments reduced the production of GM-CSF, with the PLGA-wogonin form more effective in reducing GM-CSF production compared with the soluble form (Fig. 2C). This may be because wogonin in the nanoparticle form may have a controlled release of wogonin, which may last for longer periods of time in vivo, compared with wogonin in soluble form. In blood vessels, inflammatory responses include vasodilation, enhanced permeability of vessels and blood stasis $(1,4)$. The production of inflammatory cytokines in the blood vessels may result in cytoskeletal alterations in the endothelial cells, which may eventually disrupt and increase the permeability of vessels (30). At the beginning of inflammation in blood vessels, the inflammatory cells (monocytes, neutrophils, lymphocytes and macrophages) respond by activating a number of different inflammatory pathways and promoting the production of cytokines $(5,28,30)$. Macrophages are one of major resources for producing inflammatory cytokines (30). The present study illustrated that regulating macrophage functions with wogonin reduced the production of inflammatory cytokines. This indicated 
A<smiles>COc1c(O)cc(O)c2c(=O)cc(-c3ccccc3)oc12</smiles>

B

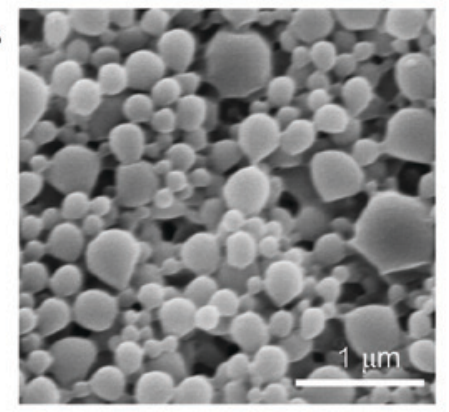

C

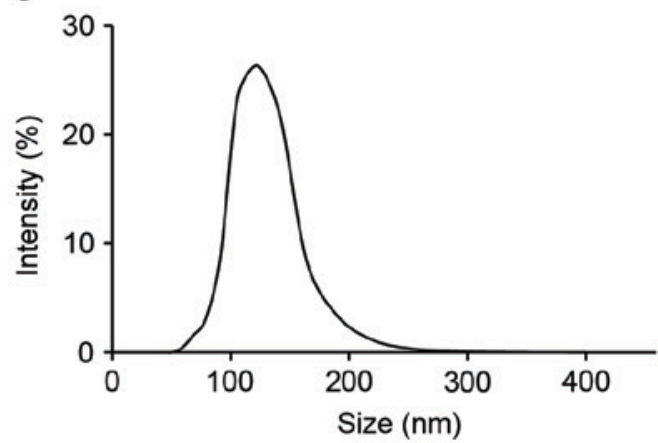

Figure 1. Characterization of biodegradable particles carrying wogonin. (A) The chemical structure of wogonin. Wogonin is poorly soluble in aqueous solution, thus using a biomaterial carrier can improve its delivery effect. (B) Scanning electron microscopy imaging of poly(lactic-co-glycolic) acid particles carrying wogonin. The particles were not very uniform in size; a feature of the emulsion synthesis technique. (C) Dynamic light scattering analysis indicated the size distribution of the nanoparticles.
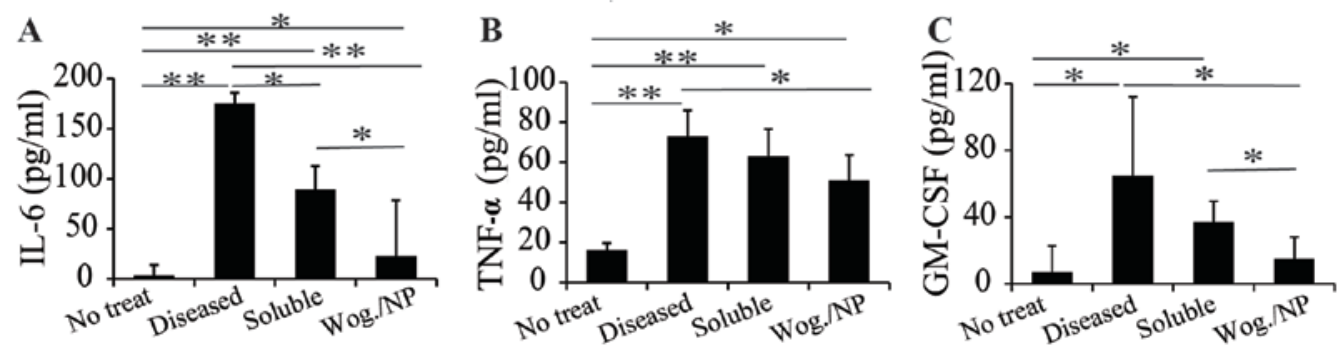

Figure 2. ELISA analysis of the levels of cytokines in mice serum. Each mouse was treated with $275 \mu \mathrm{g}$ daily wogonin or received no treatment. Peripheral blood was collected from mice for testing the level of cytokines in serum. ELISA was performed at $96 \mathrm{~h}$ post-treatment to determine the levels of (A) IL-6, (B) TNF- $\alpha$ and (C) GM-CSF. ${ }^{*} \mathrm{P}<0.05$ and ${ }^{* *} \mathrm{P}<0.01$. GM-CSF, granulocyte macrophage colony-stimulating factor; IL, interleukin; NP, PLGA nanoparticle; TNF, tumor necrosis factor; Wog, wogonin.

that the vascular inflammation, especially the inflammation induced by the cytokines produced by macrophages, can be controlled by using wogonin. Comparing the soluble wogonin and wogonin carried in PLGA nanoparticles, the cargo carried in PLGA nanoparticles (Wog./NP) was more effective due to the improved functionality of the biomaterial carrier.

The effects of wogonin on macrophage functions were also examined. Macrophages were collected from the bone marrow of untreated mice or mice induced with disease (i.e., diseased) and incubated with the different forms of wogonin in a range of doses. Macrophages obtained from diseased mice were treated with 10,25 or $100 \mu \mathrm{g} / \mathrm{ml}$ wogonin to test the effect of the cargo on cellular viability (Fig. 3A). The results demonstrated that wogonin in nanoparticle form did not significantly affect macrophage viability over $48 \mathrm{~h}$ incubation (Fig. 3A). By contrast, macrophages treated with soluble wogonin had a slightly lower viability when the dose of wogonin was increased, although no statistical difference was identified (Fig. 3A). The macrophages from diseased mice also maintains viability comparable to untreated mice, indicating the disease induction did not affect macrophage viability in mice (Fig. 3A.). The ratio of M1 macrophage cells to M2 macrophage cells was also examined; following streptozotocin-induced vascular inflammation, there was a higher M1/M2 ratio compared with Control ( $\mathrm{P}<0.05$; Fig. $3 \mathrm{~B})$. Wogonin treatments in particle form (Fig. 3B; gray bar) were more effective led to a slightly higher ratio of M1 macrophages compared to mice receiving soluble wogonin (Fig. 3B; orange bar). The M1/M2 ratio indicated the level of inflammation; M1 macrophages are responsible for the inflammatory response, whereas M2 macrophages are in involved in tissue repair and regeneration (31-33). The present results also revealed that the concentration of wogonin treatment regulated the relative ratio of M1 macrophages. In both soluble and nanoparticle wogonin treatments, the relative level of M1 macrophages decreased as the dose increased, which indicated that wogonin was able to downregulate macrophage function in a dose-dependent manner. The current study illustrated that wogonin can change the relative ratio of M1 and M2 macrophages. More studies are needed to illustrate the mechanism by which wogonin affects macrophages differentiation. That M1 macrophage level decreased with increased wogonin dose demonstrated that wogonin controls vascular inflammation by regulating the relative ratio of $\mathrm{M} 1$ and $\mathrm{M} 2$ macrophages.

The activation of macrophage surface markers by wogonin treatments was also examined. Macrophages were stimulated with LPS to promote the inflammatory response. The cells were treated for $24 \mathrm{~h}$ and collected for analysis. Expression levels of all three macrophage activation surface markers, $\mathrm{CD}^{+} 0^{+}, \mathrm{CD} 40^{+}$and $\mathrm{CD}^{+} 6^{+}$, significantly increased upon LPS treatment (Fig. 4A-C, respectively). Both soluble and particulate wogonin treatments reduced the expression of $\mathrm{CD}^{+} 0^{+}$(Fig. 4A); compared to soluble wogonin, wogonin loaded in nanoparticles was more effective in reducing the 
A

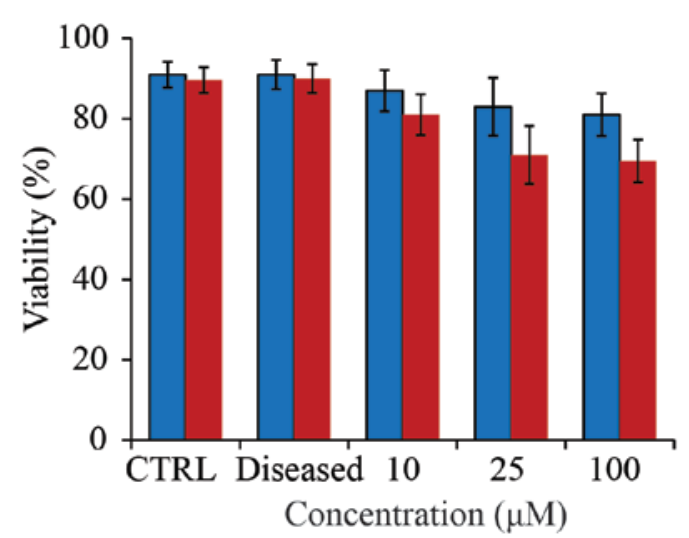

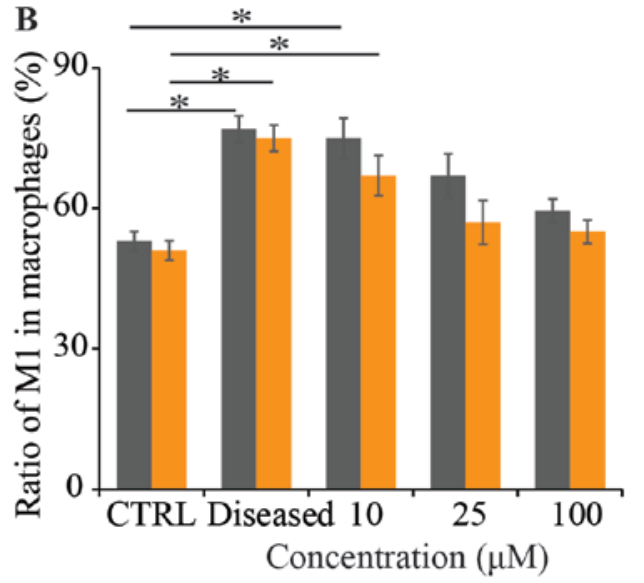

Figure 3. Effects of wogonin on macrophage viability and phenotype. (A) The effects of soluble wogonin (red bars) and wogonin carried in polymeric nanoparticles (blue bars) on macrophage viability. The cells were cultured with different concentration of wogonin for $48 \mathrm{~h}$ prior to viability testing. (B) The ratio of M1 macrophages to M2 macrophages were tested $48 \mathrm{~h}$ after treatment. The cells that received soluble wogonin treatment are indicated by the orange bars, and the cells that received wogonin nanoparticle treatment are indicated by the grey bars. ${ }^{*} \mathrm{P}<0.05$. CTRL, control.
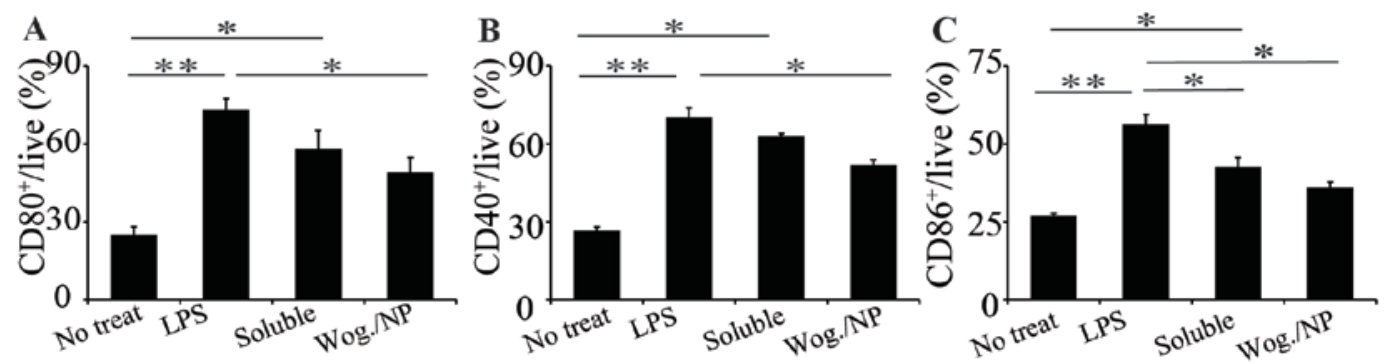

Figure 4. The effects of wogonin treatment on macrophage surface activation markers. A total of 4 groups of cells were used in the experiments: i) No treatment; ii) LPS $(1 \mu \mathrm{g} / \mathrm{ml})$; iii) LPS $(1 \mu \mathrm{g} / \mathrm{ml})+$ soluble wogonin $(45 \mu \mathrm{g} / \mathrm{ml})$; and iv) LPS $(1 \mu \mathrm{g} / \mathrm{ml})+$ wogonin loaded in nanoparticles $(45 \mu \mathrm{g} / \mathrm{ml})$. Wogonin carried in nanoparticles decreased the activation (A) $\mathrm{CD} 80^{+},(\mathrm{B}) \mathrm{CD} 40^{+}$and $(\mathrm{C}) \mathrm{CD} 86^{+}$markers on the macrophage surface. ${ }^{*} \mathrm{P}<0.05$ and ${ }^{* *} \mathrm{P}<0.01$. LPS, lipopolysaccharide; NP, nanoparticle; Wog, wogonin.
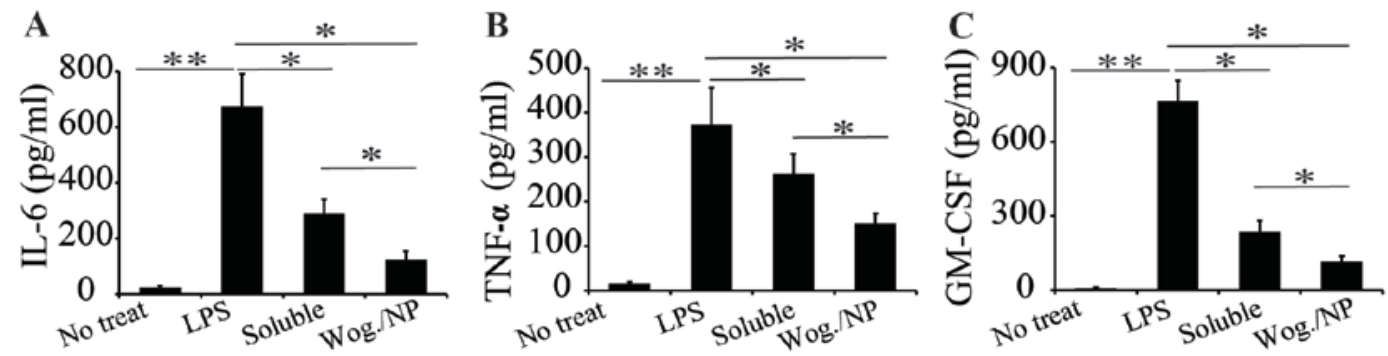

Figure 5. Secretion levels of cytokines from macrophages co-cultured with LPS and wogonin. A total of 4 groups of cells were used in the experiments: i) No treat; ii) LPS $(1 \mu \mathrm{g} / \mathrm{ml})$; iii) LPS $(1 \mu \mathrm{g} / \mathrm{ml})+$ soluble wogonin $(45 \mu \mathrm{g} / \mathrm{ml})$; and iv) LPS $(1 \mu \mathrm{g} / \mathrm{ml})+$ wogonin loaded in nanoparticles $(45 \mu \mathrm{g} / \mathrm{ml})$. The cells were treated for $60 \mathrm{~h}$, and the supernatants were collected for analyzing the level of cytokines, including (A) IL-6, (B) TNF- $\alpha$ and (C) GM-CS. ${ }^{*}<0.05$ and ${ }^{* *} \mathrm{P}<0.01$. GM-CSF, granulocyte macrophage colony-stimulating factor; IL, interleukin; NP, nanoparticle; TNF, tumor necrosis factor; Wog, wogonin.

expression of $\mathrm{CD} 80^{+}$markers. A similar trend was observed in the expression of $\mathrm{CD} 40^{+}$and $\mathrm{CD} 86^{+}$markers (Fig. 4B and $\mathrm{C}$, respectively). These results indicated that wogonin reduced the activation of macrophage markers, and wogonin loaded in PLGA was more effective compared with soluble wogonin. This was probably because the PLGA polymer carrier is biodegradable both in vitro and in vivo, releasing wogonin cargo continuously over time and providing the cells with consistent treatment; instead, soluble formulation had less efficacy as wogonin did not dissolve very well in culture medium. Wogonin has low solubility in aqueous solutions, and this is one of the advantages of using a biomaterials carrier to deliver wogonin. The present study demonstrated that wogonin can reduce macrophage surface marker expression and so reduce macrophage activation in the case of vascular inflammation. A previous study indicated that wogonin exhibits anti-inflammation functions by inhibiting vascular hyperpermeability and expression of CAMs (6). The results from the present study explain the regulation of the roles of wogonin in vascular inflammation. 
To connect the activation of macrophage surface markers with their inflammatory immune responses, the cell culture supernatant was collected and analyzed for the secretion of inflammatory cytokines, including IL-6, TNF- $\alpha$ and GM-CSF, from the macrophages in the different treatment groups (Fig. 5A-C, respectively). Upon LPS stimulation, the expression level of each inflammatory cytokine was significantly increased (Fig. 5). Both soluble and particle forms of wogonin co-treatment significantly reduced the production of IL-6 (Fig. 5A); wogonin in nanoparticle form appeared to be more potent in reducing IL- 6 production compared with wogonin in soluble form. Similarly, TNF- $\alpha$ and GM-CSF expression levels were significantly reduced in macrophages co-treated with either form of wogonin compared with LPS-only (Fig. 5B and $\mathrm{C}$, respectively). In both cases, nanoparticle wogonin was more effective in reducing the expression of these cytokines compared with the soluble form; this is probably due to the use of a particle carrier, which improved the bioavailability of wogonin to the cells, thus providing better therapeutic effects. Previous studies focused on using free wogonin for treating different diseases including inflammation (7-10). The present study demonstrated that the use of a PLGA carrier can improve the efficacy by changing the bioavailability of wogonin. As to the anti-inflammatory functions of wogonin, a previous study indicated that wogonin can regulate the production of IL-6 gene, another important inflammatory cytokine (8). Another study demonstrated that wogonin can inhibit toll-like receptor 4 and MARK inflammatory pathways (10). Compared to these existing studies, the current study confirmed the anti-IL6 functions of wogonin. It also demonstrated that wogonin can regulate another two inflammatory cytokines, TNF- $\alpha$ and GM-CSF. This result indicated that other anti-inflammatory pathways might be also involved in wogonin treatment, which will probably be the subject of our future studies.

In conclusion, The present study examined the effects of wogonin in regulating immune responses in vascular inflammation. In the in vivo experiments, wogonin downregulated the level of inflammatory cytokines. Wogonin loaded in the biomaterial carrier had an improved therapeutic effect compared with that in soluble form. The results also revealed that wogonin did not affect macrophage viability, but did regulate the relative ratio of M1 macrophages to M2 macrophages. In addition, wogonin regulated the expression of macrophage surface activation markers $\mathrm{CD}^{+} 6^{+}, \mathrm{CD} 80^{+}$and $\mathrm{CD}_{40}{ }^{+}$. Analysis of the level of inflammatory cytokines in the cell culture supernatant demonstrated that wogonin was able to inhibit the production of inflammatory cytokines from the stimulated macrophages. The current study demonstrated both inflammatory cytokines and macrophage activation were involved in the use of wogonin against vascular inflammation. Future wogonin therapy against vascular inflammation may benefit from the use of a biodegradable carrier, which can improve the therapeutic efficacy by improved bio-availability and other advantages brought by a degradable carrier, including targeting and controlled release.

\section{Acknowledgements}

This study was supported by The Shandong Provincial Hospital Affiliated to Shandong University (Jinan, China).

\section{References}

1. Virdis A and Schiffrin EL: Vascular inflammation: A role in vascular disease in hypertension? Curr Opin Nephrol Hypertens 12: 181-187, 2003.

2. Tedgui A and Mallat Z: Anti-inflammatory mechanisms in the vascular wall. Circ Res 88: 877-887, 2001.

3. Taube A, Schlich R, Sell H, Eckardt K and Eckel J: Inflammation and metabolic dysfunction: Links to cardiovascular diseases. Am J Physiol Heart Circ Physiol 302: H2148-H2165, 2012.

4. Schenkein HA and Loos BG: Inflammatory mechanisms linking periodontal diseases to cardiovascular diseases. J Clin Periodontol 40 (Suppl 14): S51-S69, 2013.

5. Ait-Oufella H, Taleb S, Mallat Z and Tedgui A: Recent advances on the role of cytokines in atherosclerosis. Arterioscler Thromb Vasc Biol 31: 969-979, 2011.

6. Xiao JR, Do CW and To CH: Potential therapeutic effects of baicalein, baicalin, and wogonin in ocular disorders. J Ocul Pharmacol Ther 30: 605-614, 2014.

7. Lee W, Ku SK and Bae JS: Anti-inflammatory effects of Baicalin, Baicalein, and Wogonin in vitro and in vivo. Inflammation 38: 110-125, 2015.

8. Zhao Y, Yao J, Wu XP, Zhao L, Zhou YX, Zhang Y, You QD, Guo QL and Lu N: Wogonin suppresses human alveolar adenocarcinoma cell A549 migration in inflammatory microenvironment by modulating the IL-6/STAT3 signaling pathway. Mol Carcinog 54 (Suppl 1): E81-E93, 2015.

9. Lee YM, Cheng PY, Chen SY, Chung MT and Sheu JR: Wogonin suppresses arrhythmias, inflammatory responses, and apoptosis induced by myocardial ischemia/reperfusion in rats. J Cardiovasc Pharmacol 58: 133-142, 2011.

10. Chen S, Xiong J, Zhan Y, Liu W and Wang X: Wogonin inhibits LPS-induced inflammatory responses in rat dorsal root ganglion neurons via inhibiting TLR4-MyD88-TAK1-mediated NF- $\kappa \mathrm{B}$ and MAPK signaling pathway. Cell Mol Neurobiol 35: 523-531, 2015.

11. Chen LG, Hung LY, Tsai KW, Pan YS, Tsai YD, Li YZ and Liu YW: Wogonin, a bioactive flavonoid in herbal tea, inhibits inflammatory cyclooxygenase-2 gene expression in human lung epithelial cancer cells. Mol Nutr Food Res 52: 1349-1357, 2008.

12. Chen YC, Shen SC, Lee WR, Lin HY, Ko CH, Shih CM and Yang LL: Wogonin and fisetin induction of apoptosis through activation of caspase 3 cascade and alternative expression of p21 protein in hepatocellular carcinoma cells SK-HEP-1. Arch Toxicol 76: 351-359, 2002.

13. Lee WR, Shen SC, Lin HY, Hou WC, Yang LL and Chen YC: Wogonin and fisetin induce apoptosis in human promyeloleukemic cells, accompanied by a decrease of reactive oxygen species, and activation of caspase 3 and $\mathrm{Ca}^{2+}$-dependent endonuclease. Biochem Pharmacol 63: 225-236, 2002.

14. Li DR, Hou HX, Zhang W and Li L: Effects of wogonin on inducing apoptosis of human ovarian cancer A2780 cells and telomerase activity. Ai Zheng 22: 801-805, 2003.

15. Wang W, Guo QL, You QD, Zhang K, Yang Y, Yu J, Liu W, Zhao L, Gu HY, Hu Y, et al: The anticancer activities of wogonin in murine sarcoma S180 both in vitro and in vivo. Biol Pharm Bull 29: 1132-1137, 2006.

16. Lin CC, Kuo CL, Lee MH, Lai KC, Lin JP, Yang JS, Yu CS, Lu CC, Chiang JH, Chueh FS, et al: Wogonin triggers apoptosis in human osteosarcoma U-2 OS cells through the endoplasmic reticulum stress, mitochondrial dysfunction and caspase-3-dependent signaling pathways. Int J Oncol 39: 217-224, 2011.

17. Lee JY and Park W: Anti-Inflammatory effect of wogonin on RAW 264.7 mouse macrophages induced with polyinosinic-polycytidylic acid. Molecules 20: 6888-6900, 2015.

18. Wakabayashi I and Yasui K: Wogonin inhibits inducible prostaglandin $\mathrm{E}_{2}$ production in macrophages. Eur J Pharmacol 406: 477-481, 2000.

19. Shieh DE, Liu LT and Lin CC: Antioxidant and free radical scavenging effects of baicalein, baicalin and wogonin. Anticancer Res 20: 2861-2865, 2000.

20. Lim BO: Efficacy of wogonin in the production of immunoglobulins and cytokines by mesenteric lymph node lymphocytes in mouse colitis induced with dextran sulfate sodium. Biosci Biotechnol Biochem 68: 2505-2511, 2004.

21. Chi YS, Lim H, Park H and Kim HP: Effects of wogonin, a plant flavone from Scutellaria radix, on skin inflammation: In vivo regulation of inflammation-associated gene expression. Biochem Pharmacol 66: 1271-1278, 2003. 
22. Yao J, Pan D, Zhao Y, Zhao L, Sun J, Wang Y, You QD, Xi T, Guo QL and Lu N: Wogonin prevents lipopolysaccharide-induced acute lung injury and inflammation in mice via peroxisome proliferator-activated receptor gamma-mediated attenuation of the nuclear factor- $\kappa B$ pathway. Immunology 143: 241-257, 2014.

23. Cohen-Sela E, Chorny M, Koroukhov N, Danenberg HD and Golomb G: A new double emulsion solvent diffusion technique for encapsulating hydrophilic molecules in PLGA nanoparticles. J Control Release 133: 90-95, 2009.

24. Ozcelikay AT, Pekiner C, Ari N, Ozturk Y, Ozuari A and Altan VM: The effect of vanadyl treatment on vascular responsiveness of streptozotocin-diabetic rats. Diabetologia 37: $572-578,1994$

25. Nasri S, Roghani M, Baluchnejadmojarad T, Rabani $\mathrm{T}$ and Balvardi M: Vascular mechanisms of cyanidin-3-glucoside response in streptozotocin-diabetic rats. Pathophysiology 18: 273-278, 2011.

26. Weischenfeldt J and Porse B: Bone marrow-derived macrophages (BMM): Isolation and applications. CSH Protoc 2008, 2008.

27. Huang AY, Golumbek P, Ahmadzadeh M, Jaffee E, Pardoll D and Levitsky $\mathrm{H}$ : Role of bone marrow-derived cells in presenting MHC class I-restricted tumor antigens. Science 264: 961-965, 1994.
28. Kim S, Lee Y, Park H, Hong D, Khang G and Lee D: Reduced inflammatory responses to poly(lactic-co-glycolic acid) by the incorporation of hydroxybenzyl alcohol releasing polyoxalate. Macromol Res 19: 1242-1249, 2011.

29. Nicolete R, dos Santos DF and Faccioli LH: The uptake of PLGA micro or nanoparticles by macrophages provokes distinct in vitro inflammatory response. Int Immunopharmacol 11: 1557-1563, 2011.

30. Dinh QN, Drummond GR, Sobey CG and Chrissobolis S: Roles of inflammation, oxidative stress, and vascular dysfunction in hypertension. Biomed Res Int 2014: 406960, 2014.

31. Martinez FO and Gordon S: The M1 and M2 paradigm of macrophage activation: Time for reassessment. F1000Prime Rep 6: 13, 2014.

32. Italiani P and Boraschi D: From monocytes to M1/M2 macrophages: Phenotypical vs. functional differentiation. Front Immunol 5: 514, 2014

33. Graziano F, Vicenzi E and Poli G: Plastic restriction of HIV-1 replication in human macrophages derived from M1/M2 polarized monocytes. J Leukoc Biol 100: 1147-1153, 2016. 